\title{
Pair correlations, short-range order, and dispersive excitations in the quasi-kagome quantum magnet volborthite
}

\author{
G. J. Nilsen, ${ }^{1,2,3, *}$ F. C. Coomer, ${ }^{3, \dagger}$ M. A. de Vries, ${ }^{4}$ J. R. Stewart, ${ }^{5}$ P. P. Deen, ${ }^{6}$ A. Harrison,,${ }^{3,7}$ and H. M. Rønnow ${ }^{1}$ \\ ${ }^{1}$ Laboratory for Quantum Magnetism, ICMP, Ecole Polytchnique Fédérale de Lausanne (EPFL), Switzerland \\ ${ }^{2}$ Institute for Solid State Physics, University of Tokyo, Kashiwa, Chiba 277-8581, Japan \\ ${ }^{3}$ School of Chemistry, University of Edinburgh, West Mains Road, Edinburgh, EH9 3JJ, United Kingdom \\ ${ }^{4}$ Department of Physics and Astronomy, University of Leeds, Leeds, LS2 9JT, United Kingdom \\ ${ }^{5}$ ISIS facility, Rutherford Appleton Laboratory, STFC, Chilton, Didcot, OX11 ODE, United Kingdom \\ ${ }^{6}$ European Spallation Source, ESS AB, P.O. Box 176, SE-22100 Lund, Sweden \\ ${ }^{7}$ Institut Laue-Langevin, 6 rue Jules Horowitz, FR-38042 Grenoble, France
}

(Received 26 August 2011; published 8 November 2011)

\begin{abstract}
We present spatial and dynamic information on the $s=1 / 2$ distorted kagome antiferromagnet volborthite, $\mathrm{Cu}_{3} \mathrm{~V}_{2} \mathrm{O}_{7}(\mathrm{OD})_{2} \cdot 2 \mathrm{D}_{2} \mathrm{O}$, obtained by polarized and inelastic neutron scattering. The instantaneous structure factor, $S(Q)$, is dominated by nearest-neighbor pair correlations, with short-range order at wave vectors $Q_{1}=$ $0.65(3) \AA^{-1}$ and $Q_{2}=1.15(5) \AA^{-1}$ emerging below $5 \mathrm{~K}$. The excitation spectrum, $S(Q, \omega)$, reveals two steep branches dispersing from $Q_{1}$ and $Q_{2}$, and a flat mode at $\omega_{f}=5.0(2) \mathrm{meV}$. The results allow us to identify the crossover at $T^{*} \sim 1 \mathrm{~K}$ in ${ }^{51} \mathrm{~V}$ NMR and specific-heat measurements as the buildup of correlations at $Q_{1}$. We compare our data to theoretical models proposed for volborthite, and also demonstrate that the excitation spectrum can be explained by spin-wave-like excitations with anisotropic exchange parameters, as suggested by recent local-density calculations.
\end{abstract}

DOI: 10.1103/PhysRevB.84.172401

PACS number(s): 75.10.Jm, 75.10.Kt, 78.70.Nx

The quantum kagome Heisenberg antiferromagnet (QKHAF) is among the most coveted targets in the quest for experimental realizations of quantum spin liquid ground states. The two most prominent physical realizations of the QKHAF studied to date are the naturally occurring minerals herbertsmithite, $\mathrm{Cu}_{3} \mathrm{Zn}(\mathrm{OH})_{6} \mathrm{Cl}_{2}$ (Ref. 1) and volborthite, $\mathrm{Cu}_{3} \mathrm{~V}_{2} \mathrm{O}_{7}(\mathrm{OH})_{2} \cdot 2 \mathrm{H}_{2} \mathrm{O}$. $^{2}$ The physics of herbertsmithite is arguably influenced by depletion of the kagome lattice caused by antisite mixing, possibly resulting in a valence bond glass state. ${ }^{3,4}$ In volborthite, the kagome planes are slightly distorted, but the lattice coverage is essentially complete. Since both systems deviate from the pure QKHAF model, focus has shifted to the intriguing question of which states arise when the QKHAF is perturbed. Remarkably, a multitude of different states have been proposed theoretically, depending on the nature of the perturbation. These range from ordered states ${ }^{5-9}$ to the aforementioned valence bond glass. Experimentally, the ground state of volborthite remains enigmatic, despite over ten years of intensive study.,10-13 Here we present a neutron-scattering investigation of volborthite, employing both $x y z$-polarized and inelastic time-of-flight techniques.

Volborthite contains distorted kagome planes of edgesharing $\mathrm{Cu}^{2+}$ octahedra, well separated $(\sim 7.2 \AA)$ by pyrovanadate columns $\left(\mathrm{V}_{2} \mathrm{O}_{7}\right)$, Fig. 1(a). There are two crystallographically distinct $\mathrm{Cu}^{2+}$ ions: $\mathrm{Cu}(2)$ forms chains along the $b$ direction, while $\mathrm{Cu}(1)$ populates the interchain sites. Locally, $\mathrm{Cu}(1)$ and $\mathrm{Cu}(2)$ reside in tetragonally and axially distorted octahedra, respectively, suggesting the $d_{3 z^{2}-r^{2}}$ orbital being singly occupied on the $\mathrm{Cu}(1)$ site, with $d_{x^{2}-y^{2}}$ the magnetically active orbital for $\mathrm{Cu}(2)$. As a consequence, there are two different nearest-neighbor exchange pathways, $J_{1}\left(r_{\mathrm{Cu}(2)-\mathrm{Cu}(2)}=2.93 \AA\right)$ and $J_{1}^{\prime}\left(r_{\mathrm{Cu}(1)-\mathrm{Cu}(2)}=3.03 \AA\right) . J_{1}$ links $\mathrm{Cu}(2)$ ions along the $b$ axis, while $J_{1}^{\prime}$ connects $\mathrm{Cu}(1)$ and $\mathrm{Cu}(2)$ ions. Furthermore, the edge sharing of the $\mathrm{Cu}(2)$ octahedra along the $b$ direction implies the possibility of a strong next-nearest-neighbor exchange, $J_{2}$.

Despite a large estimated average nearest neighbor coupling $J_{\text {avg }}=\left(2 J_{1}^{\prime}+J_{1}\right) / 3=84 \mathrm{~K}$, magnetic susceptibility and specific-heat measurements on volborthite show no signs of long-range order down to $1.8 \mathrm{~K}^{2}$. At yet lower temperatures, muon spin rotation $(\mu \mathrm{SR})$ and ${ }^{51} \mathrm{~V}$ NMR (Refs. 10-12) indicate slowing down of fluctuations at $T^{*}=1 \mathrm{~K}$, but with dynamics persisting to $20 \mathrm{mK}$. The state below $T^{*}$ was interpreted as either incommensurate or short-range correlated. Consistent with this, low-temperature specific-heat studies indicate a high density of low-energy modes below $T^{*} .{ }^{13}$

These results, however, have provided sparse insight into the nature of the magnetic correlations and excitations. Neutron scattering is an ideal probe for investigating these aspects, but has thus far not been employed, mainly due to the large background generated by spin-incoherent scattering from ${ }^{1} \mathrm{H}$ and ${ }^{51} \mathrm{~V}$. We minimized this problem by replacing most of the ${ }^{1} \mathrm{H}(\sim 98 \%)$ by ${ }^{2} \mathrm{D}$, which was achieved by performing the synthesis ${ }^{2}$ using deuterated reagents in an atmosphere of $\mathrm{N}_{2}$, followed by annealing the product several times in $\mathrm{D}_{2} \mathrm{O}$ at $95{ }^{\circ} \mathrm{C}$. Phase purity of the resulting sample was verified by powder $\mathrm{X}$-ray diffraction. The concentration of paramagnetic defects, proportional to the magnitude of the Curie tail, ${ }^{14}$ was estimated to be $<1 \%$. Polarized diffuse neutron scattering was performed on D7 at Institut Laue-Langevin (ILL) using $35.7 \mathrm{~g}$ of sample and incident energy $E_{i}=8.5 \mathrm{meV}$. Three orthogonal neutron polarizations ( $x, y$, and $z$ ) and their corresponding spin-flip and non-spin-flip cross sections were analyzed, which allowed for isolation of the magnetic scattering cross section, $(d \sigma / d \Omega)_{\text {mag. }}{ }^{15}$ As no energy analysis was used, the observed scattering was effectively integrated up to $\omega=8.2 \mathrm{meV}$, thus approximating the instantaneous structure factor, $S(Q)$. Spectra were measured at $T=200,15,10$, and $5 \mathrm{~K}$ (Fig. 2). At 


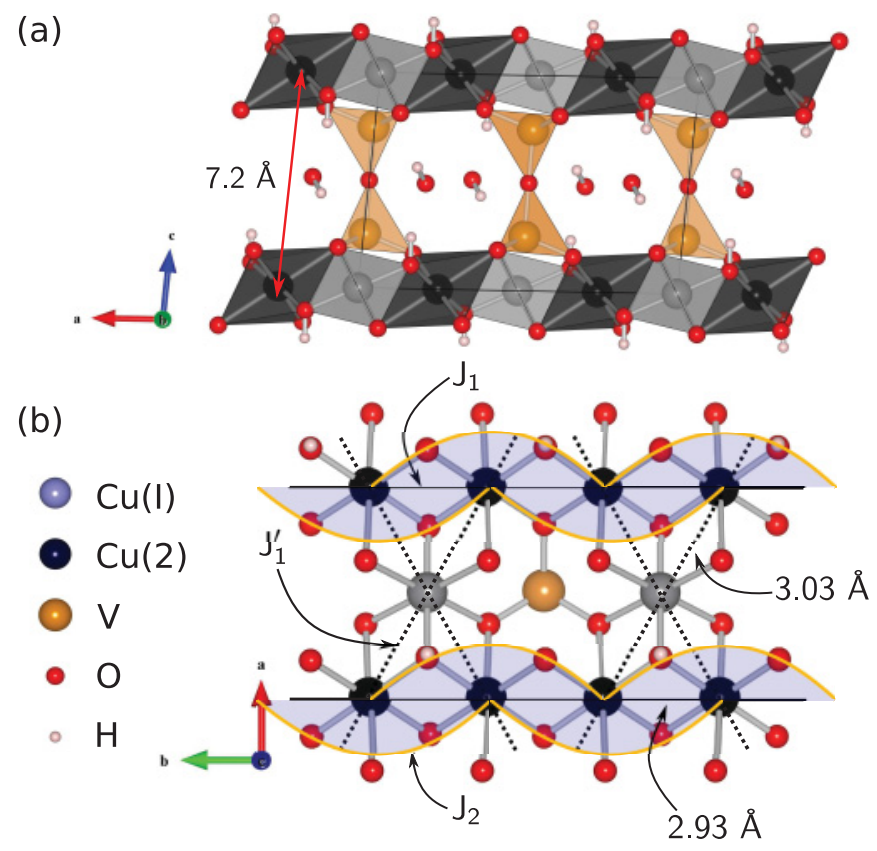

FIG. 1. (Color online) Structure of volborthite. (a) Kagome planes of $\mathrm{Cu}(1)$ and $\mathrm{Cu}(2)$ octahedra (respectively, gray and black) are separated by $\mathrm{V}_{2} \mathrm{O}_{7}$ columns (orange). (b) Local environments of $\mathrm{Cu}^{2+}$ in the quasi-kagome plane. Solid and dashed black lines indicate the two nearest-neighbor exchanges, $J_{1}$ and $J_{1}^{\prime}$. The next-nearestneighbor exchange $J_{2}$ along the $b$ direction is shown in orange (gray) arcs.

$200 \mathrm{~K} \sim 2 J$, the $Q$ dependence of $(d \sigma / d \Omega)_{\text {mag }}$ approximately follows the $\mathrm{Cu}^{2+}$ form factor, $|f(Q)|^{2}$, as anticipated for a paramagnet. Reducing the temperature to $15 \mathrm{~K}$, broad diffuse scattering develops around $Q=1.1-1.4 \AA^{-1}$. The $Q$ dependence is consistent with a buildup of nearest-neighbor pair correlations, described by the powder-averaged structure factor $(d \sigma / d \Omega)_{\mathrm{mag}}$ :

$$
\begin{aligned}
\left(\frac{d \sigma}{d \Omega}\right)_{\mathrm{mag}}= & \frac{2}{3}\left(\frac{\gamma_{n} r_{0}}{2} \mu\right)^{2}|f(Q)|^{2} \\
& \times\left(1+Z_{1}\left\langle\mathbf{S}_{0} \cdot \mathbf{S}_{1}\right\rangle \frac{\sin Q r}{Q r}\right),
\end{aligned}
$$

where the second term in parentheses reflects the average correlation $\left\langle\mathbf{S}_{0} \cdot \mathbf{S}_{1}\right\rangle=-0.25(5)$ between a unit spin and its $Z_{1}$ nearest neighbors at a distance $r_{\mathrm{Cu}-\mathrm{Cu}} \sim 3 \AA$. The total scattering was found to be $0.99(8) \mu_{B}^{2}$ per $\mathrm{Cu}$, corresponding to $33 \%$ of the full $g^{2} S(S+1)$. The fact that correlations are weak and confined to only nearest neighbors even at $T / J_{\text {avg }} \sim 0.2$ are both indicators of strong frustration in volborthite.

As $T$ is further decreased to first $10 \mathrm{~K}$ and then $5 \mathrm{~K}$, the broad diffuse scattering persists, but some (14\%) of the spectral weight is shifted into two sharper (although not resolution limited) peaks at $Q_{1}=0.65(3) \AA^{-1}$ and $Q_{2}=1.15(5) \AA^{-1}$. The corresponding correlation length, $\xi=24(8) \AA \sim 8 r_{\mathrm{Cu}-\mathrm{Cu}}$, was extracted by fitting the diffuse scattering to Eq. (1) and the two sharper features to Lorentzians [Fig. 2(d)].

The dynamical structure factor, $S(Q, \omega)$, was investigated by inelastic neutron scattering on IN4 at ILL. Spectra were
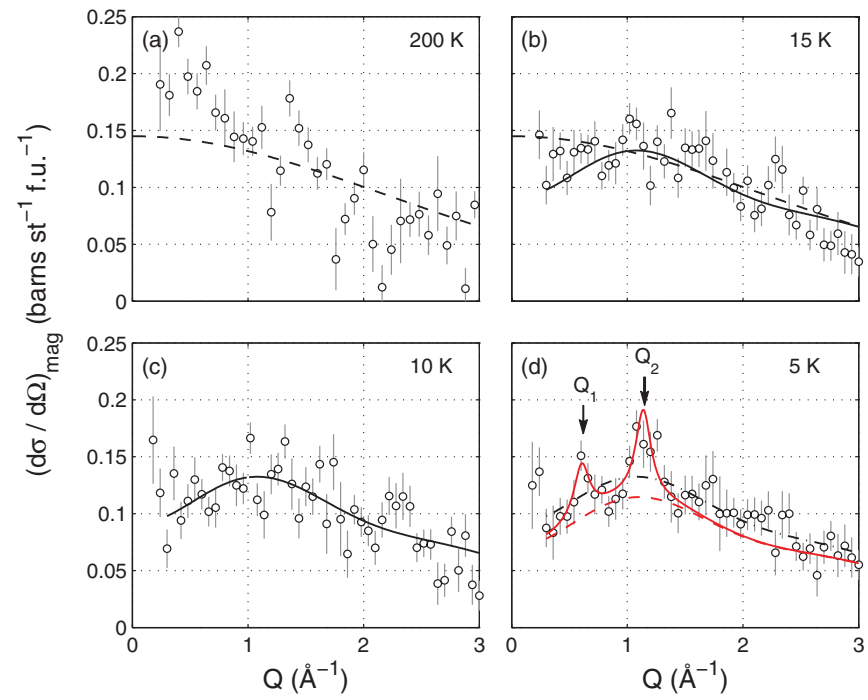

FIG. 2. (Color online) $(d \sigma / d \Omega)_{\mathrm{mag}}$ for volborthite at $T=$ $200,15,10$, and $5 \mathrm{~K}$. (a) $(d \sigma / d \Omega)_{\text {mag }}$ mimics $f(Q)^{2}$ (dashed black line) for $\mathrm{Cu}^{2+}$. [(b),(c)] Spectral weight shifts into a broad feature around 1.1-1.4 $\AA^{-1}$. Solid line is fit to Eq. (1). (d) Fit to $(d \sigma / d \Omega)_{\mathrm{mag}}$ at $5 \mathrm{~K}$ as described in the text (red, gray). The dashed black line indicates the fit at $10 \mathrm{~K}$, showing the shift of spectral weight into the two sharp features at $Q_{1}$ and $Q_{2}$.

collected using incident energy $E_{i}=17.2 \mathrm{meV}$, giving an elastic $Q$ range of $0.65-4.95 \AA^{-1}$. Further experiments were carried out on MARI at ISIS $\left(E_{i}=15 \mathrm{meV}, 0.45-4.95 \AA^{-1}\right.$ at $\omega=0 \mathrm{meV}$ ). Data are summarized in Fig. 3. At $50 \mathrm{~K} \sim J / 2$, $S(Q, \omega)$ shows only a broad response centered at $Q=1.1 \AA^{-1}$ and extending to $5 \mathrm{meV}$, consistent with fluctuations in a short-range-correlated system. The $Q$ dependence extracted by integrating over the range $2-6 \mathrm{meV}$ is indicated in the top panel of Fig. 3(b). Its form is similar to $S(Q)$ at 10 and $15 \mathrm{~K}$, and can also be fitted by Eq. (1) using $r \sim 3.5(2) \AA \sim$ $r_{\mathrm{Cu}-\mathrm{Cu}}$. Acoustic phonons are observed dispersing from nuclear Bragg positions at $Q>2 \AA^{-1}$ and intense phonon scattering is found above $7 \mathrm{meV}$, making extraction of the magnetic signal at these energies difficult. Cooling to $5 \mathrm{~K}$, the low- $Q$, low- $\omega$ intensity has largely moved into two features: an intense broad flat band, centered at $\omega_{f}=5.0(2) \mathrm{meV}$, and a nearly vertical bar of scattering at $Q=1.08(2) \AA^{-1}$, which coincides with the $Q_{2}$ peak in $S(Q)$. Both of these features sharpen as the temperature is reduced toward $0.05 \mathrm{~K}$, with a second bar of scattering at $Q=0.68(4) \AA^{-1} \sim Q_{1}$ growing below $1.7 \mathrm{~K}$. $Q$ cuts through the $Q_{1}$ and $Q_{2}$ modes are shown in the lower four panels of Fig. 3(b). While the peaks narrow somewhat with decreasing $T$, a more dramatic change is observed in their respective intensities, $I\left(Q_{1}\right)$ and $I\left(Q_{2}\right)$. On cooling, $I\left(Q_{2}\right)$ remains constant, while $I\left(Q_{1}\right)$ increases to a final ratio $I\left(Q_{1}\right) / I\left(Q_{2}\right)=1.6$ at $0.05 \mathrm{~K}$. The buildup of dynamical correlations at the $Q_{1}$ position thus coincides with the transition at $T^{*}$ observed in NMR and $\mu \mathrm{SR}$.

The line shape and amplitude of the flat mode, on the other hand, show little temperature dependence, with only slight narrowing to become resolution limited between 5 and $1.7 \mathrm{~K}$. Such narrow flat modes are often associated with two-level excitations, e.g., between a singlet and triplet. Indeed, such 

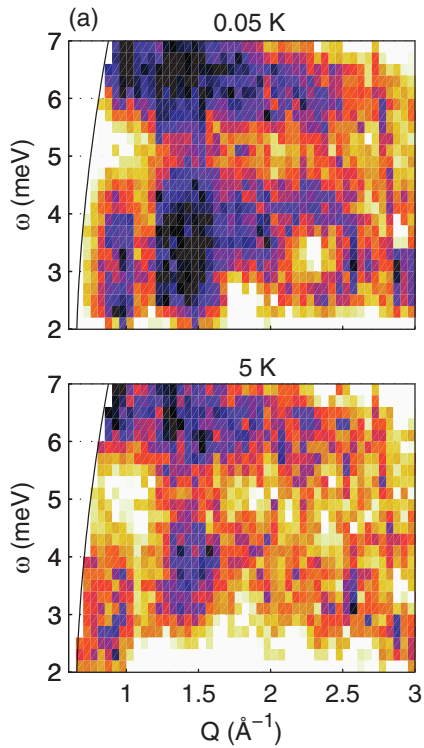
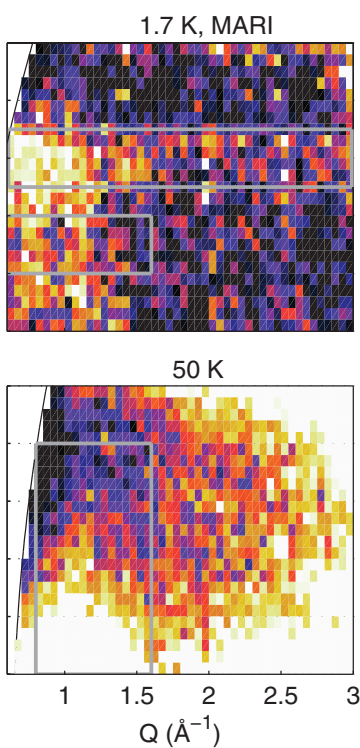
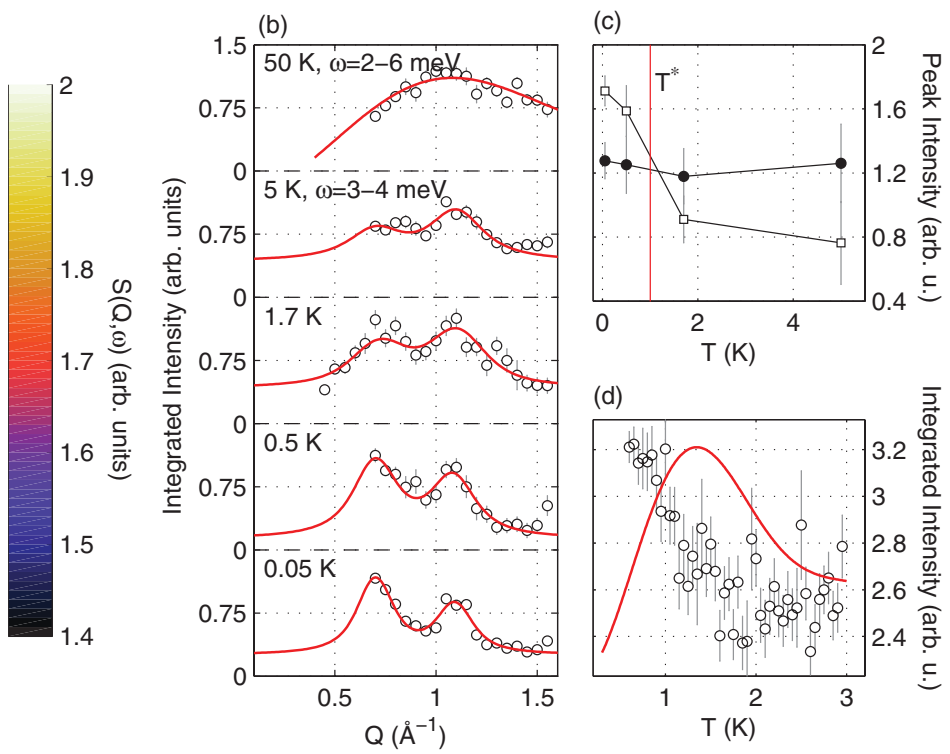

FIG. 3. (Color online) (a) $S(Q, \omega)$ from IN4 at $T=0.05,5$, and $50 \mathrm{~K}$, and MARI at $1.7 \mathrm{~K}$. The integration ranges used for the cuts in panels (b) and (d) are shown as gray rectangles. (b) $Q$ dependence of intensity over energy ranges and 3-4 meV for $0.05-5 \mathrm{~K}$ (fit by two resolution-convoluted Lorentzians) and $2-6 \mathrm{meV}$ for $50 \mathrm{~K}$ [fit by Eq. (1)]. (c) Temperature variation of intensities of the $Q_{1}$ (open circles) and $Q_{2}$ (closed circles) modes. $T^{*}=1 \mathrm{~K}$ is indicated by a vertical line. (d) $Q$ dependence of the flat mode integrated over $4.5-5.5 \mathrm{meV}$. $S(Q)$ for a singlet-triplet excitation is shown by the solid line.

excitations are expected in the site-diluted QKHAF., ${ }^{46}$ While the predominantly nearest-neighbor correlations observed in
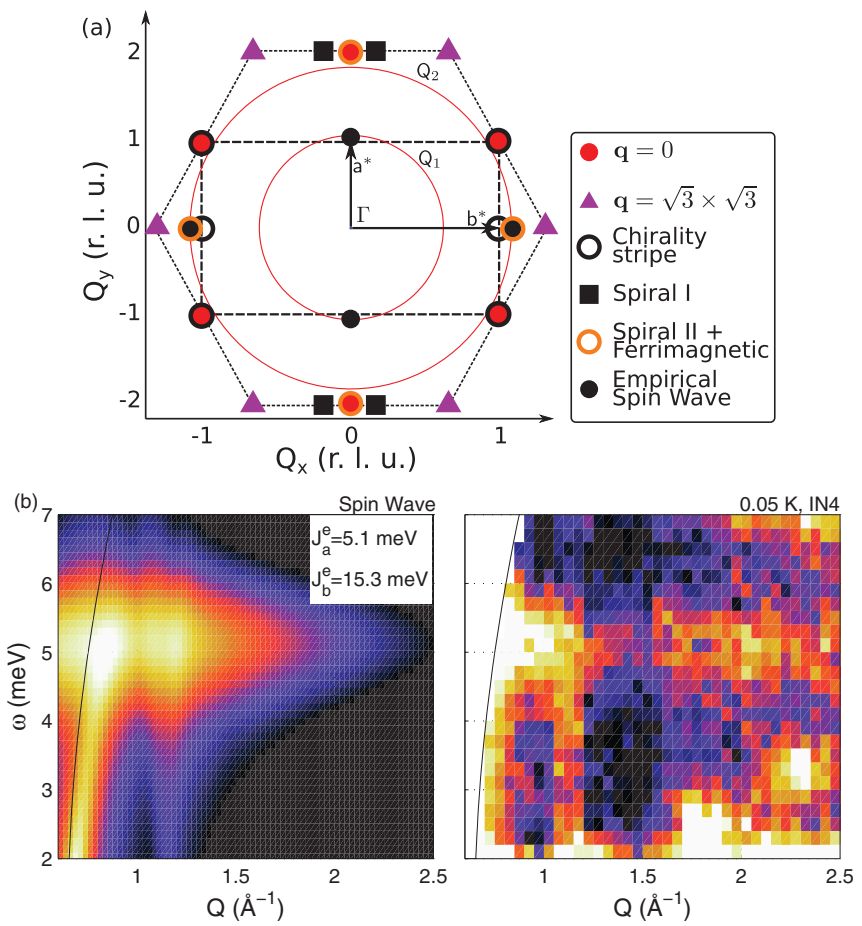

FIG. 4. (Color online) (a) Reciprocal space of volborthite: the structural unit cell is indicated by the dashed rectangle, and the extended Brillouin zone of the kagome lattice by the dotted hexagon. Symbols represent the strongest Bragg peaks of the orders listed in the text. (b) The experimental $S(Q, \omega)$ measured at $0.05 \mathrm{~K}$ compared with the powder-averaged $S(Q, \omega)$ derived from our empirical spin-wave model. The solid line in the left panel indicates the $(Q, \omega)$ window of the experiment.
$S(Q)$ are consistent with such a state, the $Q$ dependence of the flat mode does not match the singlet-triplet $S(Q)^{3,4}$ [Fig. 3(d)]. Another possible explanation for the flat band is thus that it is associated with the short-range order observed at the $Q_{1}$ and $Q_{2}$ positions. This leads to two scenarios: (i) that the ground state possesses a degeneracy which results in a flat band, as is the case in the ground state of the classical kagome systems, ${ }^{17}$ or (ii) that the flat band arises as a consequence of powder averaging at the zone boundary of a spin-wave dispersion.

In the pure QKHAF, no short-range order is expected, contrary to observations. To understand the low- $T$ short-rangeordered state, it is therefore necessary to look theoretically further afield. From structural considerations outlined in the introduction, the Hamiltonian of volborthite includes at least three exchange terms $\left(J_{1}, J_{1}^{\prime}\right.$, and $\left.J_{2}\right)$ as well as a Dzyaloshinskii-Moriya (DM) interaction, for which the dominant component is considered to be $D_{z}$. The subsets of this model which have been treated semiclassically or quantum mechanically are $J_{1}=J_{1}^{\prime}>0, J_{2}=0$, and $D_{z} \neq 0$, the isotropic kagome DM model (IKDM) ( $^{7,8,18}$ and $J_{1} \neq$ $J_{1}^{\prime}>0, J_{2}=0$, and $D_{z}=0$, the anisotropic kagome (AK) model. $^{5,6}$ In addition, recent $\mathrm{L}(\mathrm{S}) \mathrm{DA}+U$ calculations have suggested a model where $-J_{1}^{\prime} / J_{1} \sim 1.2-2,-J_{2} / J_{1}=$ $1.1-1.6$, and $J_{1}^{\prime}=8.6 \mathrm{meV}$. This model, which we call the coupled chain (CC) model, has only been treated classically so far. ${ }^{19}$ The five possible ordered states which emerge from these models are $\mathbf{q}=0$ order (IKDM for $D_{z}>0.1$, AK for $\left.1 / 2<J_{1}^{\prime} / J_{1}<1\right), \mathbf{q}=\sqrt{3} \times \sqrt{3}\left(\mathrm{AK}\right.$ for $\left.1<J_{1} / J_{1}^{\prime}<1.3\right)$, chirality stripe (AK for $J_{1} / J_{1}^{\prime}>1.3$ ), ferrimagnetic (AK for $J_{1}^{\prime} / J_{1}<1 / 2$, CC for $J_{2}<\left|J_{1}\right| / 4+J_{1}^{\prime} / 8$ ), and two kinds of spiral, spiral I (CC for $J_{2}>\left|J_{1}\right| / 4+J_{1}^{\prime} / 8$ ) or spiral II [AK for $J_{1}^{\prime} \ll J_{1}$ (Ref. 20)]. The positions of strong magnetic Bragg peaks for these structures in the reciprocal plane of the kagome lattice are compared to our experimental $Q_{1}$ and $Q_{2}$ 
in Fig. 4(a). None of the proposed structures are likely to yield strong scattering on the ring in reciprocal space defined by $Q_{1}$.

Therefore, instead of attempting to describe the observed $S(Q, \omega)$ in terms of the microscopic model above, we take the phenomenological approach of constructing a generic dispersion emanating from antiferromagnetic zone centers at high-symmetry positions on the circles defined by $Q_{1}$ and $Q_{2}$ [Fig. 4(a)]. One example of such a dispersion among the scenarios considered assumes zone centers close to the (10) and (01) positions, yielding the dispersion

$\omega(\mathbf{q})=\sqrt{\left(2 J_{a}^{e}+2 J_{b}^{e}\right)^{2}-\left[2 J_{a}^{e} \cos \left(q_{x} a\right)+2 J_{b}^{e} \cos \left(q_{y} b\right)\right]^{2}}$,

where $J_{a, b}^{e}$ are effective exchanges, giving the amplitudes of the dispersion along $a$ and $b$. Then,

$$
S(\mathbf{q}, \omega)=|F(\tau)|^{2} \frac{2-\cos \left(q_{x} a\right)-\cos \left(q_{y} b\right)}{\omega(\mathbf{q})},
$$

where $|F(\tau)|^{2}$ is the structure factor at the chosen positions, $1 / \omega$ describes antiferromangetic spin-wave intensity, and the numerator is a geometric term yielding zero intensity at ferromagnetic zone centers. To yield a smooth continuous function, $S(\mathbf{q}, \omega)$ was interpolated between adjacent Brillouin zones. Finally, the spectrum was powder averaged and convoluted with the experimental resolution. The result of this procedure using $J_{a}^{e}=5.1 \mathrm{meV}$ and $J_{b}^{e}=15.3 \mathrm{meV}$ closely resembles the experimental data [Fig. 4(b)]. One important conclusion from this analysis is that the steepness of the $Q_{2}$ mode implies that the $5 \mathrm{meV}$ band cannot be the global zone boundary energy. Instead, the flat band is found to be a saddle point, which requires sizable anisotropy between $J_{a}^{e}$ and $J_{b}^{e}$. This result is consistent with $\mathrm{LS}(\mathrm{D}) \mathrm{A}+U$ calculations, which also suggest a significant anisotropy in exchange along $a$ and $b$.

In summary, we have reported polarized and inelastic neutron-scattering results on the quasi-kagome $s=1 / 2$ antiferromagnet volborthite. These reveal three important features: (i) buildup of nearest-neighbor pair correlations from 50 to $10 \mathrm{~K}$; (ii) short-range order indicated by peaks at $Q_{1}=$ $0.65(3) \AA^{-1}$ and $Q_{2}=1.15(5) \AA^{-1}$ in the diffuse and inelastic scattering below $5 \mathrm{~K}$; and (iii) dispersive modes emanating from both $Q_{1}$ and $Q_{2}$ as well as a flat mode at $\omega_{f}=5 \mathrm{meV}$. The inelastic intensity at $Q_{1}$ becomes dominant below $1.7 \mathrm{~K}$, identifying the nature of the low- $T$ state reported from ${ }^{51} \mathrm{~V}$ NMR and $C_{p}$. Most models proposed for volborthite, however, inaccurately predict no scattering at $Q_{1}$, and could therefore be ruled out. We provide an empirical dispersion model which reproduces the experimental observations for an anisotropic set of exchanges, in rough agreement with recent $\mathrm{LS}(\mathrm{D}) \mathrm{A}+U$ calculations. A quantum treatment of this model could well yield a correct description of both the correlations and excitation spectrum that we have uncovered experimentally.

We thank Z. Hiroi, B. Fåk, A. Schnyder, P. Mendels, F. Bert, and O. Janson for stimulating discussions, G. Ehlers and J. Gardner for assistance during initial experiments, and SNF for support. *gorannilsen@gmail.com

${ }^{\dagger}$ Present address: Dept. of Pure and Applied Chemistry, University of Strathclyde, Glasgow, G1 1XL UK.

${ }^{1}$ M. Shores, E. Nytko, B. Bartlett, and D. Nocera, J. Am. Chem. Soc. 127, 13462 (2005).

${ }^{2}$ Z. Hiroi, M. Hanawa, N. Kobayashi, M. Nohara, H. Takagi, Y. Kato, and M. Takigawa, J. Phys. Soc. Jpn. 70, 3377 (2001).

${ }^{3}$ M. A. de Vries, J. R. Stewart, P. P. Deen, J. O. Piatek, G. J. Nilsen, H. M. Rønnow, and A. Harrison, Phys. Rev. Lett. 103, 237201 (2009).

${ }^{4}$ R. R. P. Singh, Phys. Rev. Lett. 104, 177203 (2010).

${ }^{5}$ T. Yavors'kii, W. Apel, and H.-U. Everts, Phys. Rev. B 76, 064430 (2007).

${ }^{6}$ F. Wang, A. Vishwanath, and Y. B. Kim, Phys. Rev. B 76, 094421 (2007).

${ }^{7}$ O. Cepas, C. M. Fong, P. W. Leung, and C. Lhuillier, Phys. Rev. B 78, 140405 (2008).

${ }^{8}$ I. Rousochatzakis, S. R. Manmana, A. M. Lauchli, B. Normand, and F. Mila, Phys. Rev. B 79, 214415 (2009).

${ }^{9}$ L. Messio, C. Lhuillier, and G. Misguich, Phys. Rev. B 83, 184401 (2011).

${ }^{10}$ F. Bert, D. Bono, P. Mendels, F. Ladieu, F. Duc, J. C. Trombe, and P. Millet, Phys. Rev. Lett. 95, 087203 (2005).
${ }^{11}$ H. Yoshida, Y. Okamoto, T. Tayama, T. Sakakibara, M. Tokunaga, A. Matsuo, Y. Narumi, K. Kindo, M. Yoshida, M. Takigawa, and Z. Hiroi, J. Phys. Soc. Jpn. 78, 043704 (2009).

${ }^{12}$ M. Yoshida, M. Takigawa, H. Yoshida, Y. Okamoto, and Z. Hiroi, Phys. Rev. Lett. 103, 077207 (2009).

${ }^{13}$ S. Yamashita, T. Moriura, Y. Nakazawa, H. Yoshida, Y. Okamoto, and Z. Hiroi, J. Phys. Soc. Jpn. 79, 083710 (2010).

${ }^{14}$ F. Bert, D. Bono, P. Mendels, J. Trombe, P. Millet, A. Amato, C. Baines, and A. Hillier, J. Phys.: Condens. Matter 16, S829 (2004).

${ }^{15}$ J. R. Stewart, P. P. Deen, K. H. Andersen, H. Schober, J. F. Barthelemy, J. M. Hillier, A. P. Murani, T. Hayes, and B. Lindenau, J. Appl. Crystallogr. 42, 69 (2009).

${ }^{16} \mathrm{~S}$. Dommange, M. Mambrini, B. Normand, and F. Mila, Phys. Rev. B 68, 224416 (2003).

${ }^{17}$ F. C. Coomer, A. Harrison, G. S. Oakley, J. Kulda, J. R. Stewart, J. A. Stride, B. Fak, J. W. Taylor, and D. Visser, J. Phys.: Condens. Matter 18, 8847 (2006).

${ }^{18}$ L. Messio, O. Cepas, and C. Lhuillier, Phys. Rev. B 81, 064428 (2010).

${ }^{19}$ O. Janson, J. Richter, P. Sindzingre, and H. Rosner, Phys. Rev. B 82, 104434 (2010).

${ }^{20}$ A. P. Schnyder, O. A. Starykh, and L. Balents, Phys. Rev. B 78, 174420 (2008) 